\title{
Clinical outcomes of continuous vs intermittent meropenem infusion for the treatment of sepsis: A systematic review and meta-analysis
}

\author{
Peng Chen ${ }^{1, A-F}$, Fuchao Chen ${ }^{2, F}$, Jiexin Lei, ${ }^{3, F}$, Benhong Zhou ${ }^{1, F}$ \\ ${ }^{1}$ Department of Pharmacy, Renmin Hospital of Wuhan University, China \\ 2 Department of Pharmacy, Dongfeng Hospital, Hubei University of Medicine, Shiyan, China \\ ${ }^{3}$ Department of Endocrinology, Renmin Hospital of Wuhan University, China \\ A - research concept and design; B - collection and/or assembly of data; $\mathrm{C}$ - data analysis and interpretation; \\ $\mathrm{D}$ - writing the article; $\mathrm{E}$ - critical revision of the article; $\mathrm{F}$ - final approval of the article
}

\section{Address for correspondence}

Benhong Zhou

E-mail: benhongzh@whu.edu.cn

Funding sources

None declared

Conflict of interest

None declared

Received on September 22, 2018

Reviewed on October 19, 2018

Accepted on May 1, 2020

Published online on August 12, 2020

\begin{abstract}
The antibiotic meropenem is commonly administered to patients with sepsis and septic shock. The aim of this study was to conduct a meta-analysis to evaluate the clinical efficacy and safety of continuous compared to intermittent meropenem infusion for the treatment of sepsis. Electronic databases such as PubMed, EMBASE, Cochrane Library, and China National Knowledge Infrastructure (CNKI) were researched to collect clinical trials comparing continuous and intermittent infusion of meropenem in patients with sepsis. After data extraction and quality assessment of the included studies, Stata v. 12.0 software (Stata Corporation LLC, College Station, USA) was used for a meta-analysis of mortality, clinical cure, microbiological eradication, and safety. Seven studies with a total of 1,191 participants met the inclusion criteria and were included in the meta-analysis. The meta-analysis showed that continuous meropenem infusion was superior to intermittent infusion in terms of mortality (combined risk ratio $(R R)=0.66,95 \%$ confidence interval $(95 \% C l)=0.46-0.98, p=0.03)$, clinical cure rate (combined $R R=1.15,95 \% C l=1.02-1.30, p=0.026)$ and microbiological eradication (combined $\mathrm{RR}=1.20,95 \% \mathrm{Cl}=1.01-1.42, \mathrm{p}=0.04$ ), although it may increase the incidence of some adverse events (AEs). Compared with intermittent dosing, administration of meropenem antibiotics through continuous infusion in patients with sepsis is associated with decreased hospital mortality, increased clinical cure rates and greater microbiological eradication. Further high-quality studies should be conducted to confirm our findings.
\end{abstract}

Key words: sepsis, meropenem, continuous infusion
Cite as

Chen P, Chen F, Lei J, Zhou B. Clinical outcomes of continuous vs intermittent meropenem infusion for the treatment of sepsis: A systematic review and meta-analysis. Adv Clin Exp Med. 2020;29(8):993-1000. doi:10.17219/acem/121934

D0I

10.17219/acem/121934

Copyright

Copyright by Author(s)

This is an article distributed under the terms of the

Creative Commons Attribution 3.0 Unported (CC BY 3.0)

(https://creativecommons.org/licenses/by/3.0/) 


\section{Introduction}

Severe infections in critically ill patients are a major burden in the intensive care unit (ICU), with persistently high mortality rates. ${ }^{1}$ Optimized antibiotic therapy has been suggested as an intervention likely to improve treatment outcomes for critically ill patients. ${ }^{2}$ However, antibiotic resistance has become a major healthcare problem affecting morbidity and mortality in the clinical setting. Antibacterial drug discovery and development have slowed considerably in recent years. ${ }^{3} \mathrm{~W}$ ith the increase of antibiotic resistance and the decrease of the development of new anti-biological drugs, more research on existing antibiotics is needed. In recent years, the effort to maximize antibiotic activity has led to an interest in optimizing antibiotic dosing using the pharmacokinetic (PK) and pharmacodynamic (PD) principles of antibiotics. ${ }^{4}$

Due to the wide-spectrum activity against variety of Gram-negative and Gram-positive microorganisms, and good penetration of body fluids and tissues, meropenem become a common choice for the treatment of critically ill patients. ${ }^{5}$ Similar to other $\beta$-lactam antibiotics, it displays time-dependent bactericidal activity and PK/PD characteristics. The parameter that can best predict antibacterial efficacy is the percentage of the dosing interval that free drug concentrations remain above the minimum inhibitory concentration (MIC) during each dosing interval (referred to as $\% f \mathrm{~T}>\mathrm{MIC}){ }^{6,7}$ The optimal outcome of treatment of critically ill patients is most likely to occur when the PK/PD targets are achieved, which is closely related to the maximum antibiotic activity. A minimum standard for carbapenems is that $\mathrm{T}>$ MIC should be maintained at least $40 \%$, and a $\mathrm{T}>\mathrm{MIC}$ of $100 \%$ is associated with significantly better clinical and bacteriological outcomes in patients with serious bacterial infections. ${ }^{8,9}$

Pharmacokinetic studies in both non-critically ill and critically ill patients have demonstrated that administration of $\beta$-lactam antibiotics using continuous infusion results in consistent attainment of drug exposures associated with maximal antibacterial effects. ${ }^{10}$ Thus, continuous infusion of meropenem has been suggested to maximize the therapeutic potential in critically ill patients. Recently, the use of continuous administration of meropenem among patients with sepsis has been studied in some trials and indicated greater PK efficacy, bacteriological eradication and clinical cure rates. ${ }^{11,12}$ However, the efficacy and safety information of these clinical studies are not identical. Thus, the goal of our analysis was to evaluate the clinical efficacy and safety of continuous compared to intermittent meropenem infusion for the treatment of sepsis, to provide systematic clinical evidence for antibiotic therapy.

\section{Material and methods}

\section{Data sources and literature search}

We performed a systematic review and meta-analysis to compare continuous and intermittent infusion of meropenem in patients with sepsis. Two reviewers independently searched the medical literature for relevant clinical trials using the electronic databases of PubMed, Excerpta Medica (EMBASE), Cochrane Library, China National Knowledge Infrastructure (CNKI; www.cnki.net), Chinese Scientific Journals Full Text database (CSJFT), Wanfang Data Knowledge Service Platform (WKSP; www.wanfangdata.com.cn), and Chinese Biomedical Literature Service System (CBMdisc), through August 2018. This was supplemented by searching the reference lists of all retrieved studies, review articles, abstracts, and conference reports. The key words used in this search were: [Meropenem], [Antipseudomonal $\beta$-lactams], [Continuous infusion], [Prolonged infusion], [Intermittent infusion], [Short-term intravenous infusion], [Critically ill patients], and [Sepsis]. There were no language restrictions.

\section{Study selection}

Clinical trials that met the following criteria were included: 1) randomized, controlled trials (RCTs) or cohort studies; 2) prospective clinical trials of continuous compared to intermittent infusion of meropenem treatment in patients with sepsis; 3) studies with all patients enrolled fulfilling the criteria of sepsis; 4) studies reporting data on mortality, clinical cure, microbiological eradication, as well as adverse events (AEs) etc. Exclusion criteria were the following: 1) repeat studies, abstracts, letters, reviews, editorials, or comments; 2) studies reporting on the comparative outcomes of extended or continuous compared to intermittent but for different meropenem products duration in the 2 arms; 3 ) case reports and case series including $<10$ patients; or 4) studies reporting only $\mathrm{PK}$ or PD outcomes.

\section{Data extraction and quality assessment}

Two review authors independently screened the titles and abstracts of each study. The following information was extracted from each study: the first author, the year of publication, the number of patients enrolled in the study, and the therapeutic regimen and doses, in order to understand the baseline of all the included studies. A modified Jadad scale was used to assess the quality of the included randomized studies. The scores of high-quality studies ranged from 4 to 8 , whereas low-quality studies ranged from 0 to 3 . For non-randomized studies, the quality was assessed using Newcastle-Ottawa Quality Assessment Scale. Each study was graded as either low quality (0-5) or high quality (6-9). Any disagreements were resolved by the $3^{\text {rd }}$ author. 


\section{Statistical analysis}

The differences between the continuous compared to intermittent administration of meropenem were assessed using the pooled risk ratio (RR) with $95 \%$ confidence intervals (95\% CI). The summary RR assessments were conducted using a random- or fixed-effect model. Inter-study heterogeneity was tested using the Q-statistic and quantified using the $\mathrm{I}^{2}$ statistic. If $\mathrm{I}^{2}$ was $<50 \%\left(P_{\text {heterogeneity }}>0.1\right)$, the Mantel-Haenszel fixed-effect model was used; if not, the random-effect model was used. The sensitivity analyses were performed according to the risk of bias. We assessed publication bias using visual inspection of the funnel plot and Egger's test. All calculations were performed using Stata v. 12.0 software (Stata Corporation LLC, College Station, USA). The level of significance was set at $\mathrm{p}$-value less than 0.05 or 0.01 .

\section{Results}

\section{Search results}

The systematic search of the literature for trials on continuous compared to intermittent meropenem infusion for sepsis therapy produced 108 potentially relevant records from the primary search of databases. Of the studies initially identified, we excluded reports that did not fulfill our inclusion criteria after first screening of the titles and abstracts. Finally, 7 studies ${ }^{13-19}$ were considered eligible for the meta-analysis, including $1 \mathrm{RCT}^{15}$ and 6 prospective studies. ${ }^{13,14,16-19}$ A flowchart describing the trial screening and selection procedure is shown in Fig. 1. The 7 selected studies, involving a total of 1,191 patients (continuous group: 587 patients; intermittent group: 604 patients), were published between 2012 and 2018. The sample sizes of these studies ranged from 20 to 220 . The total daily dose of meropenem varied both within and between the individual studies. When reported, the duration of treatment was also a variable (Table 1). The Jadad scores of the 7 studies included in the meta-analysis are also listed in Table 1; the mean Jadad score was 4.23 (range: $3-6$ ), suggesting that the overall study quality was fair.

\section{Statistical analysis of efficacy outcomes}

\section{Mortality}

Four trials ${ }^{13,14,17,18}$ presented information analyzing mortality. Overall, the meta-analysis showed that continuous infusion of meropenem was associated with a lower mortality rate than intermittent intravenous infusion (484 patients, $\mathrm{RR}=0.66,95 \% \mathrm{CI}=0.46-0.98, \mathrm{Z}=2.17, \mathrm{p}=0.03$; Fig. 2), suggesting that the risk of death in patients with sepsis treated with continuous infusion of meropenem was $34 \%$ lower compared with patients treated with intermittent infusion, using the fixed-effects model (heterogeneity test, $\chi^{2}=1.42$, degrees of freedom $(\mathrm{df})=3(\mathrm{p}=0.702)$, $\left.\mathrm{I}^{2}=0 \%\right)$.

\section{Clinical cure rate}

The RR of the clinical cure rate was reported in all studies. ${ }^{13-19}$ Pooling the outcomes of the 7 studies showed that there was a significant statistical difference in the clinical cure rates between sepsis patients receiving continuous meropenem infusion and those receiving intermittent infusion (557 patients, $\mathrm{RR}=1.15,95 \% \mathrm{CI}=1.02-1.30$,
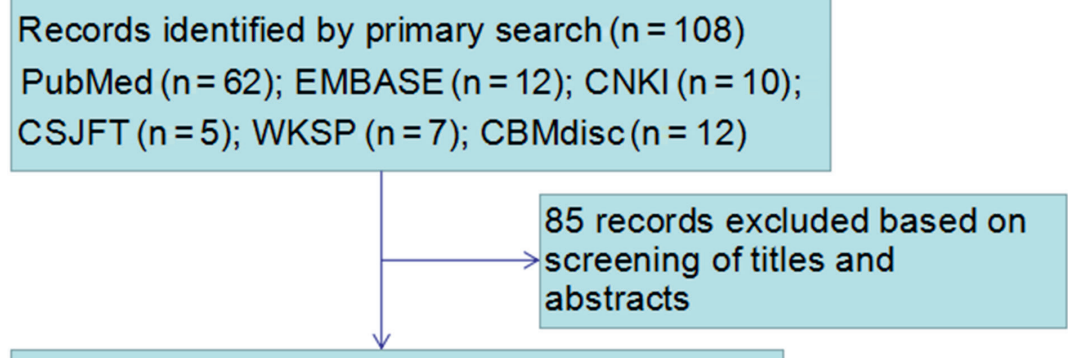

\section{3 potentially relevant full-text articles included for analysis}

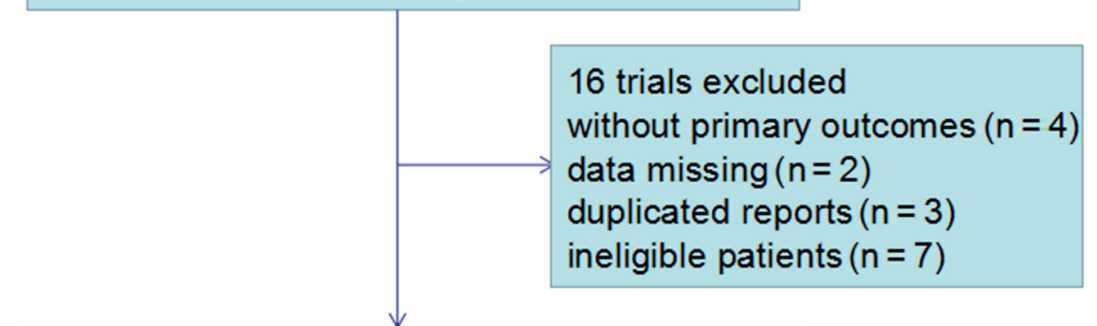

7 articles included in the meta-analysis 


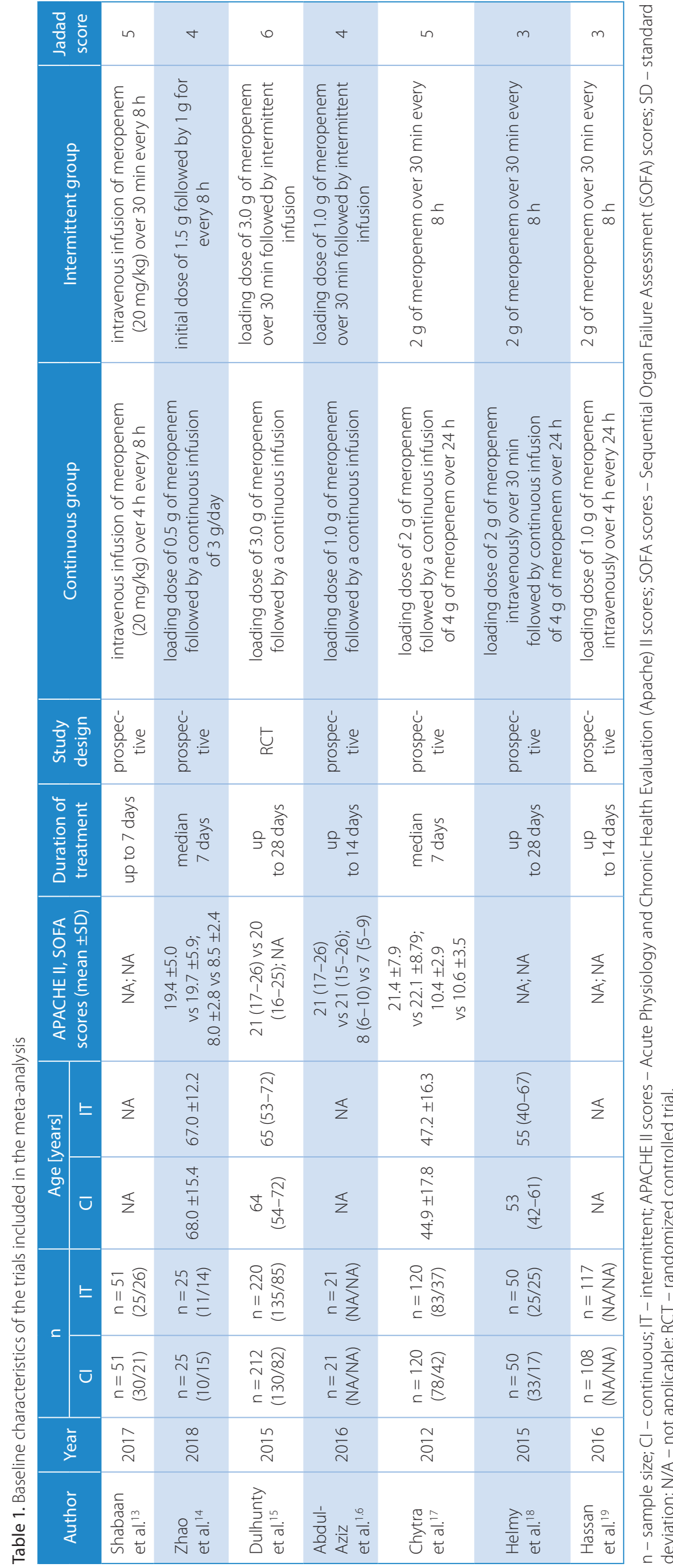

$\mathrm{Z}=2.22$, $\mathrm{p}=0.026$; Fig. 3). Heterogeneity was not observed in the studies $\left(x^{2}=4.62, \mathrm{df}=6\right.$ $\left.(\mathrm{p}=0.594), \mathrm{I}^{2}=0 \%\right)$.

\section{Microbiological eradication}

Data on comparisons of the microbiological eradication of continuous compared to intermittent intravenous administration of meropenem was reported in 4 trials. ${ }^{13,14,17,18}$ The results of our fixed-effects $\left(\mathrm{X}^{2}=3.93, \mathrm{df}=3(\mathrm{p}=0.378)\right.$, $\left.\mathrm{I}^{2}=2.9 \%\right)$ meta-analysis for microbiological eradication are summarized in Fig. 4. The results indicated that the microbiological eradication for the continuous group was significantly higher than in the intermittent group (484 patients, $\mathrm{RR}=1.20,95 \% \mathrm{CI}=1.01-1.42, \mathrm{Z}=1.78$, $\mathrm{p}=0.04 ;$ Fig. 4).

\section{Adverse events}

Three studies ${ }^{13,15,17}$ provided data regarding AEs that occurred during treatment. In 1 study, out of 212 patients in the continuous group, 39 (18.4\%) experienced AEs, compared with 53 of $220(24.1 \%)$ in the intermittent group. $.^{15} \mathrm{Ab}-$ normalities in liver and kidney function tests were reported in 1 study, in which 3 out of $51 \mathrm{pa}-$ tients (6\%) in the continuous group experienced acute kidney injury, whereas 18 of 12 patients (23.5\%) in the intermittent group experienced them. ${ }^{13}$ It has been suggested that gastrointestinal AEs (diarrhea and vomiting) are the most frequent side effects of meropenem in adults, with no significant difference between continuous infusion and intermittent administration (4.2\% with diarrhea in the constant group compared to $5.8 \%$ in the intermittent group and $1.7 \%$ experiencing vomiting in the constant group compared to $2.5 \%$ in the intermittent group). ${ }^{17}$

\section{Other outcomes of the meta- analysis}

Other clinical outcomes of continuous compared to intermittent meropenem infusion for the treatment of sepsis, such as the length of ICU stay, length of hospital stay, ICU survival, ICU-free days, etc., were reported in the included studies and are listed in Table 2.

\section{Publication bias}

We assessed publication bias using a funnel plot and Egger's test in this study (Fig. 5). The funnel plot had a certain asymmetry, indicating that 


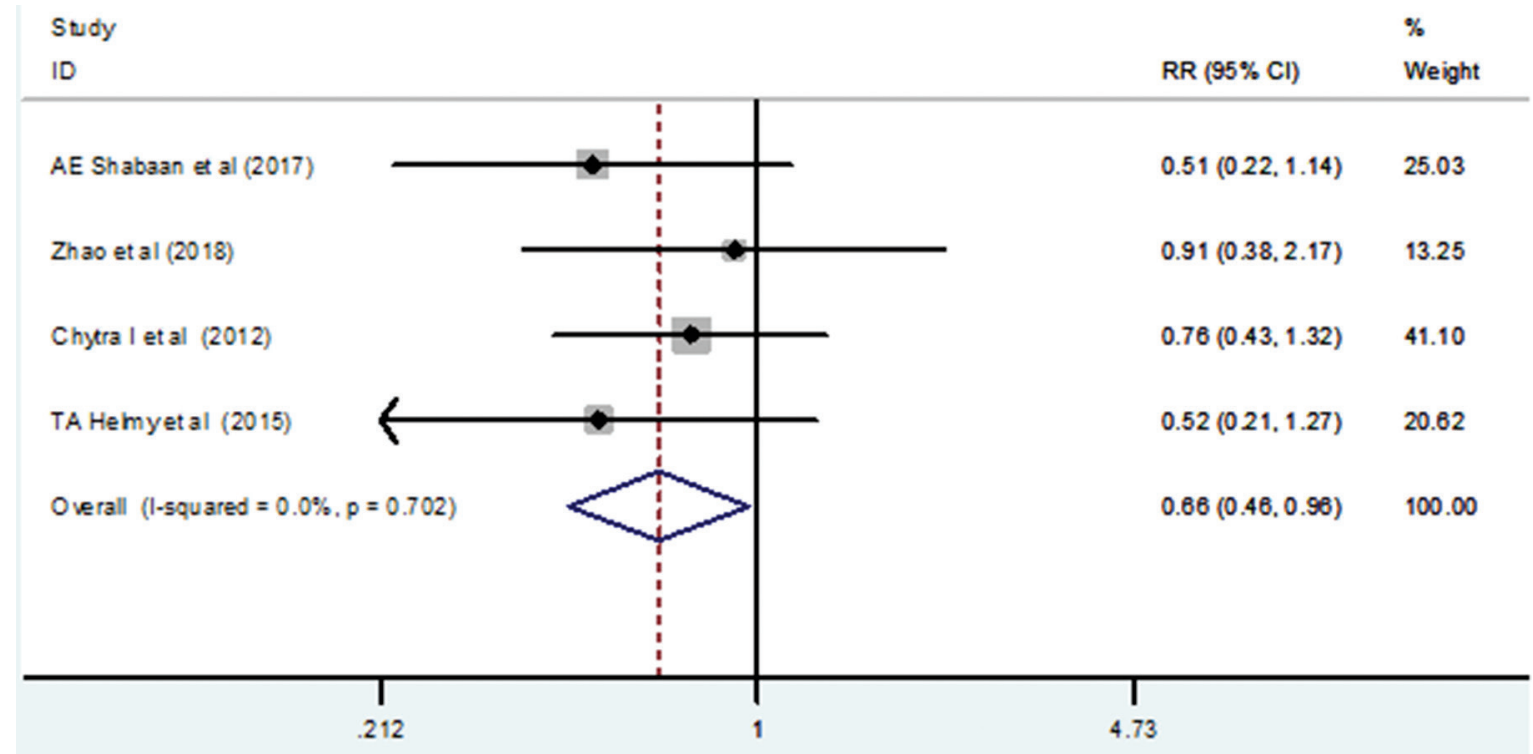

Fig. 2. Forest plot analysis of the mortality rates of continuous compared to intermittent meropenem

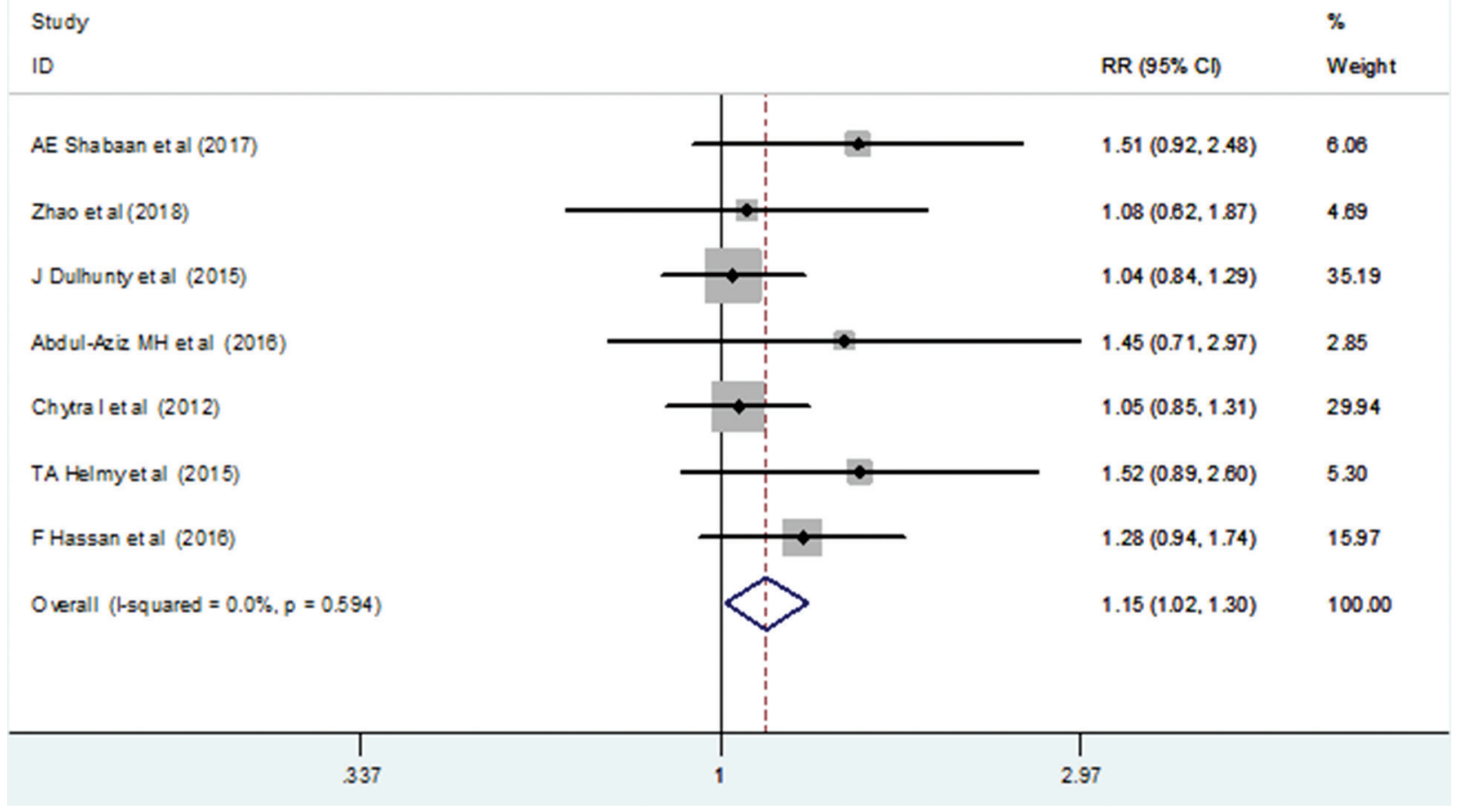

Fig. 3. Forest plots analysis of the clinical cure rates of continuous compared to intermittent meropenem

Table 2. Other outcomes of continuous compared to intermittent meropenem infusion for the treatment of sepsis

\begin{tabular}{|c|c|c|c|c|c|c|}
\hline \multirow[b]{2}{*}{ Outcomes } & \multirow[b]{2}{*}{ Studies } & \multicolumn{2}{|c|}{ Patients } & \multirow{2}{*}{$\begin{array}{l}\text { RR/WMD } \\
(95 \% \mathrm{CI})\end{array}$} & \multirow[b]{2}{*}{$\begin{array}{l}\text { Heterogeneity } \\
\qquad\left(1^{2}, P\right)\end{array}$} & \multirow[b]{2}{*}{ p-value } \\
\hline & & $\begin{array}{l}\text { continuous } \\
\text { group }\end{array}$ & $\begin{array}{l}\text { intermittent } \\
\text { group }\end{array}$ & & & \\
\hline Length of ICU stay & 3 & 166 & 166 & $-1.40(-2.19,-0.61)$ & $66 \% ; 0.65$ & 0.005 \\
\hline Hospital length of stay & 3 & 288 & 296 & $-1.87(-2.23,-1.50)$ & $41 \% ; 0.18$ & $<0.01$ \\
\hline ICU survival & 4 & 378 & 386 & $-0.30(-0.73,0.13)$ & $0 \% ; 0.54$ & 0.62 \\
\hline ICU-free days & 4 & 378 & 386 & $-0.11(-0.54,0.32)$ & $12 \% ; 0.57$ & 0.60 \\
\hline Emergence of resistance & 2 & 332 & 340 & $-16.23(-29.86,-2.59)$ & $88 \% ; 0.004$ & 0.02 \\
\hline
\end{tabular}

ICU - intensive care unit. 


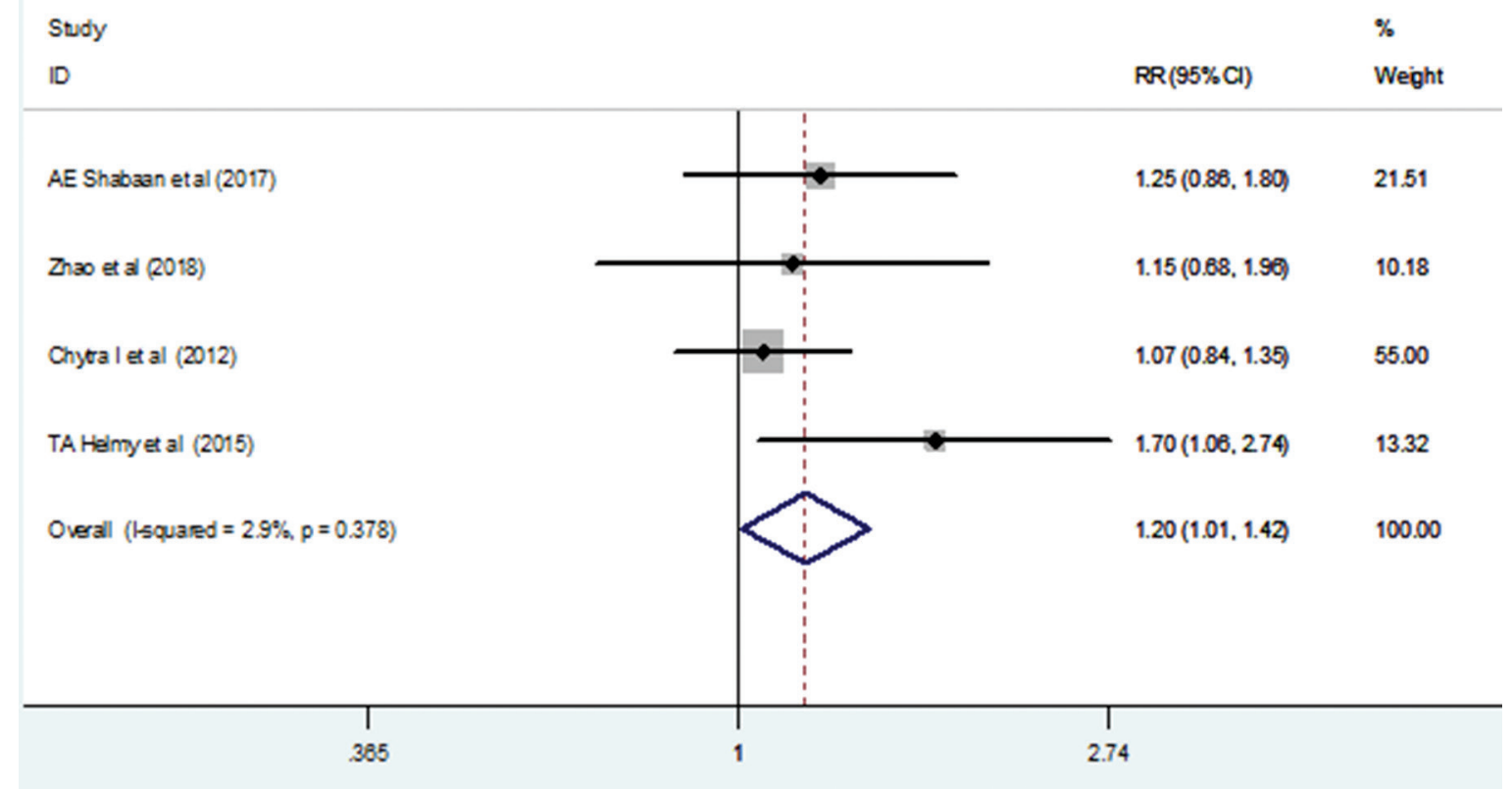

Fig. 4. Forest plot analysis of the microbiological eradication of continuous compared to intermittent meropenem

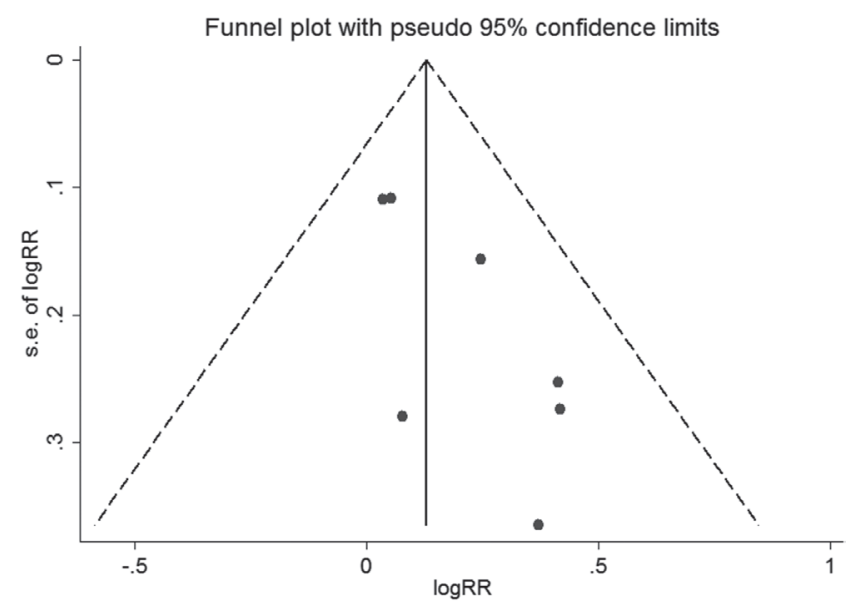

Fig. 5. Funnel plot of publication bias

there is some degree of publication bias in the literature. However, the number of studies included is small, so the funnel plot may not be convincing. Additionally, it was revealed that publication bias was not significant according to Egger's test for the incidence of AEs $(\mathrm{Z}=1.54 ; \mathrm{p}=0.41)$.

\section{Discussion}

\section{Key findings}

To our knowledge, this study is the first meta-analysis to compare outcomes of sepsis patients receiving continuous compared to intermittent intravenous meropenem. In this meta-analysis, which includes data from $1,191 \mathrm{pa}-$ tients, we found that continuous infusion of meropenem resulted in lower mortality than intermittent infusion.
Compared with intermittent infusion, continuous infusion of meropenem was associated with superior clinical cure rates, which is a more subjective outcome. ${ }^{20}$ Furthermore, a significantly higher rate of microbiological eradication was found in the continuous group compared with the intermittent group, although an insufficient number of patients or studies was included in most of these analyses. ${ }^{21}$ The findings of this meta-analysis suggest that continuous infusion of meropenem could achieve significant clinical improvement in the treatment of sepsis patients.

\section{Relationship to previous studies}

Studies of continuous infusion of $\beta$-lactam antibiotics, including meropenem, are numerous. ${ }^{22}$ These studies suggest that continuous infusion achieves a greater likelihood of achieving PK/PD targets than standard intermittent infusion in critically ill patients. ${ }^{23}$ However, the clinical value of continuous infusion with meropenem for patients with sepsis has not been systematically analyzed. It has been confirmed that sepsis patients are more likely to have pathophysiological changes leading to sub-therapeutic drug concentrations. ${ }^{24,25}$ A number of PK studies of critically ill patients with sepsis have reported that administration through continuous infusion increases the achievement of target concentrations, both in plasma and in tissues, compared with intermittent dosing. ${ }^{26}$ More important, previous metaanalyses were less selective than the present analysis in their inclusion criteria, included data from both critically ill and non-critically ill patients, and allowed different $\beta$-lactam antibiotics in the 2 treatment groups, which may have diluted any advantage of continuous infusion. ${ }^{27}$ Our meta-analysis narrowed the subject to sepsis patients and the study drug to meropenem, which could overcome the deficiencies above. 


\section{Implications of study findings}

Besides the main outcome parameters (clinical cure, mortality and microbiological eradication), there are other factors that differentiate continuous administration from intermittent administration. ${ }^{28}$ Our results showed that continuous infusion of meropenem could shorten ICU stays and total hospitalization times, which indicates that continuous administration may be a more economical therapy than intermittent administration for sepsis patients. ${ }^{29}$ Regarding safety, the most commonly reported AEs associated with continuous meropenem infusion included diarrhea, rash, seizures, nausea, and vomiting, as well as hepatic injury. ${ }^{30}$ Our study implied that administration of meropenem through continuous infusion in sepsis patients was safer compared with intermittent infusion, although the relationship with clinical cure was more complex.

\section{Other considerations}

Increasing the $\% f \mathrm{~T}>\mathrm{MIC}$ for $\beta$-lactams has been associated with increased therapeutic efficacy and delaying the emergence of resistance, and these benefits can be achieved with continuous infusion. ${ }^{31,32}$ However, theoretically speaking, carbapenems such as meropenem may be unsuitable for administration through continuous infusion due to stability issues. ${ }^{33}$ Patel et al. showed that $1 \mathrm{mg} / \mathrm{mL}$ of meropenem was stable for a longer time than $20 \mathrm{mg} / \mathrm{mL}$ and $50 \mathrm{mg} / \mathrm{mL}$ at $4-5^{\circ} \mathrm{C}$ after storage for $3-4 \mathrm{~h} .{ }^{34}$ Tomasello et al. found that there were no statistical differences in the percentage deviation values of the stability profile between concentrations of $4 \mathrm{mg} / \mathrm{mL}$ and $10 \mathrm{mg} / \mathrm{mL}$ of meropenem after 3-8 $\mathrm{h}$ when the temperature was controlled at $25^{\circ} \mathrm{C} .{ }^{35}$ Katip et al. demonstrated that $10 \mathrm{mg} / \mathrm{mL}$ meropenem solution was stable (maintained more than $90 \%$ of its initial concentration) for up to $10 \mathrm{~h}$ at $25^{\circ} \mathrm{C}$, and that $20 \mathrm{mg} / \mathrm{mL}$ meropenem solution was stable for $6 \mathrm{~h}$ at $25^{\circ} \mathrm{C} .{ }^{36}$ More importantly, meropenem is only stable for $8-12 \mathrm{~h}$ at room temperature, thus casting doubts on any potential benefit of continuous delivery. ${ }^{37}$ This is an important issue in tropical countries where meropenem concentrations decreased by $4 \%$ and $12 \%$ when stored at room temperature for $3 \mathrm{~h}$ and $8 \mathrm{~h}$, respectively, although 24-hour stability can be maintained if meropenem temperature is kept below $4^{\circ} \mathrm{C} .^{38}$

\section{Strengths and limitations}

There are several limitations in this meta-analysis that should be considered when interpreting the data. First, the number of studies and patients included in this study is small, which will make the conclusion less reliable. Second, differences in treatment regimens and doses of drugs add to the clinical heterogeneity in the data. Third, the criteria used in most trials for the definition and severity of sepsis are not in accordance to the current definitions.
Finally, publication bias might have occurred, and it might not be completely reflected by funnel plot. Therefore, additional large-scale, high-quality, placebo-controlled, double-blind trials are needed to confirm our findings.

\section{Conclusions}

The evidence from mainly non-randomized studies suggests that continuous infusion of meropenem could lead to superior treatment outcomes, including mortality, clinical cure, microbiological eradication, and AEs. However, well-designed RCTs are warranted to validate these findings before they can be widely applied in clinical practice.

\section{ORCID iDs}

Benhong Zhou (D) https://orcid.org/0000-0002-6129-0182

Peng Chen (D) https://orcid.org/0000-0003-4936-2064

\section{References}

1. Hidalgo F, Mas D, Rubio M, Garcia-Hierro P. Infections in critically ill burn patients. Med Intensiva. 2016;40(3):179-185.

2. McKinney CW, Pruden A. Ultraviolet disinfection of antibiotic resistant bacteria and their antibiotic resistance genes in water and wastewater. Environ Sci Technol. 2012;46(24):13393-13400.

3. Liu YF, Yan JJ, Lei HY, et al. Loss of outer membrane protein C in Escherichia coli contributes to both antibiotic resistance and escaping antibody-dependent bactericidal activity. Infect Immun. 2012;80(5): 1815-1822.

4. Wiklund S, Fagerberg I, Örtqvist $\AA$, et al. Knowledge and understanding of antibiotic resistance and the risk of becoming a carrier when travelling abroad: A qualitative study of Swedish travelers. Scand J Public Health. 2015;43(3):302-308.

5. Tiberi S, Payen MC, Sotgiu G, et al. Effectiveness and safety of meropenem/clavulanate-containing regimens in the treatment of MDR-and XDR-TB. Eur Respir J. 2016;47(4):1235-1243.

6. Afshartous D, Bauer SR, Connor MJ, et al. Pharmacokinetics and pharmacodynamics of imipenem and meropenem in critically ill patients treated with continuous venovenous hemodialysis. Am J Kidney Dis. 2014;63(1):170-171.

7. Chalhoub H, Sáenz $Y$, Rodriguezvillalobos H, et al. High-level resistance to meropenem in clinical isolates of Pseudomonas aeruginosa in the absence of carbapenemases: Role of active efflux and porin alterations. Int J Antimicrob Agents. 2016;48(6):740-743.

8. Laishram S, Anandan S, Devi BY, et al. Determination of synergy between sulbactam, meropenem and colistin in carbapenem-resistant Klebsiella pneumoniae and Acinetobacte rbaumannii isolates and correlation with the molecular mechanism of resistance. J Chemother. 2016;28(4):297-303.

9. Payen MC, Muylle I, Vandenberg O, et al. Meropenem-clavulanate for drug-resistant tuberculosis: A follow-up of relapse-free cases. Int J Tuberc Lung Dis. 2018;22(1):34-39.

10. Cheatham SC, Fleming MR, Healy DP, et al. Steady-state pharmacokinetics and pharmacodynamics of meropenem in morbidly obese patients hospitalized in an intensive care unit. J Clin Pharmacol. 2014; 54(3):324-330.

11. Lenhard JR, Bulitta JB, Connell TD, et al. High-intensity meropenem combinations with polymyxin B: New strategies to overcome carbapenems resistance in Acinetobacter baumannii. J Antimicrob Chemother. 2017;72(1):153-165.

12. McCarthy MW, Walsh TJ. Meropenem/vaborbactam fixed combination for the treatment of patients with complicated urinary tract infections. Drugs Today (Barc). 2017;53(10):521-530.

13. Shabaan AE, Nour I, Eldegla HE, Nasef H, Shouman B, Abdel-Hady H. Conventional versus prolonged infusion of meropenem in neonates with Gram-negative late onset sepsis: A randomized controlled trial. Pediatr Infect Dis J. 2017;36(4):358-363. 
14. Zhao HY, Gu J, Jie L, et al. Pharmacokinetic and pharmacodynamic efficacies of continuous versus intermittent administration of meropenem in patients with severe sepsis and septic shock: A prospective randomized pilot study. Chin Med J (Eng). 2017;130(10):1139-1145.

15. Dulhunty JM, Roberts JA, Davis JS, et al; BLING II Investigators for the ANZICS Clinical Trials Group. A multicenter randomized trial of continuous versus intermittent $\beta$-lactam infusion in severe sepsis. Am J Respir Crit Care Med. 2015;192(11):1298-1305.

16. Abdul-Aziz MH, Sulaiman $\mathrm{H}$, Mat-Nor MB, et al. Beta-Lactam Infusion in Severe Sepsis (BLISS): A prospective, two-centre, open-labelledrandomised controlled trial of continuous versus intermittent betalactam infusion in critically ill patients with severe sepsis. Intensive Care Med. 2016;42(10):1535-1545.

17. Chytra I, Stepan M, Benes J, et al. Clinical and microbiological efficacy of continuous versus intermittent application of meropenem in critically ill patients: A randomized open-label controlled trial. Crit Care. 2012;16(3):R113-R116.

18. Helmy TA, Abdelghaffar AA, Fathy EM, et al. Continuous versus intermittent intravenous meropenem in severe sepsis. IJPBS. 2015;5:4457.

19. Fahmi H, Noorizan AA, Yahaya H, Hazlinda AH. Clinical outcomes of critically III patients with sepsis receiving extended and standardmeropenem infusion in Malaysian hospitals. Int J Pharm Pharm Sci. 2016;10:S12S12.

20. Yusuf E, Spapen H, Pierard D. Prolonged vs intermittent infusion of piperacillin/tazobactam in critically ill patients: A narrative and systematic review. J Crit Care. 2014;29(6):1089-1095.

21. Chu DC, Mehta AB, Walkey AJ. Practice patterns and outcomes associated with procalcitonin use in critically ill patients with sepsis. Clin Infect Dis. 2017;64(11):1509-1515.

22. Zielske J, Bohne S, Brunkhorst FM, Axer H, Guntinas-Lichius O. Acute and long-term dysphagia in critically ill patients with severe sepsis: Results of a prospective controlled observational study. Eur Arch Otorhinolaryngol. 2014;271(11):3085-3093.

23. Del BV, Giacobbe DR, Marchese A, et al. Meropenem for treating KPCproducing Klebsiella pneumoniae bloodstream infections: Should we get to the PK/PD root of the paradox? Virulence. 2016;8(1):66-73.

24. Kristoffersson AN, David-Pierson P, Parrott NJ. Simulation-based evaluation of PK/PD indices for meropenem across patient groups and experimental designs. Pharm Res. 2016;33(5):1115-1125.

25. Vardakas KZ, Voulgaris GL, Maliaros A, Samonis G, Falagas ME. Prolonged versus short-term intravenous infusion of antipseudomonal $\beta$-lactams for patients with sepsis: A systematic review and metaanalysis of randomised trials. Lancet Infect Dis. 2018;18(1):108-120.
26. Dong $\mathrm{H}$, Zhong Y. Response to: Continuous versus intermittent infusion of vancomycin in adult patients: A systematic review and metaanalysis. Int J Antimicrob Agents. 2016;48(1):114-115.

27. Yang $\mathrm{H}$, Zhang $\mathrm{C}$, Zhou Q, Wang $\mathrm{Y}$, Chen L. Clinical outcomes with alternative dosing strategies for piperacillin/tazobactam: A systematic review and meta-analysis. PLoS One. 2016;10(1):274-289.

28. Sato $Y$, Sandoh $M$, Hanaki $H$, et al. Evaluation of usefulness and PK-PD analysis of meropenem in children with various infections. Gan To Kagaku Ryoho. 2012;60(3):335-341.

29. Delfino E, Fucile C, Del VB, et al. Pharmacokinetics of high-dose extended-infusion meropenem during pulmonary exacerbation in adult cystic fibrosis patients: A case series. New Microbiol. 2018;41(1): 47-51.

30. Sjövall F, Alobaid AS, Wallis SC, Perner A, Lipman J, Roberts JA. Maximally effective dosing regimens of meropenem in patients with septic shock. J Antimicrob Chemother. 2018;73(1):191-198.

31. Shotwell MS, Nesbitt R, Madonia PN, et al. Pharmacokinetics and pharmacodynamics of extended infusion versus short infusion piperacillin-tazobactam in critically ill patients undergoing CRRT. Clin J Am Soc Nephrol. 2016;11(8):1377-1383.

32. Cojutti PG, Barbarino C, De AM, Hope W, Pea F. Higher than standard meropenem and linezolid dosages needed for appropriate treatment of an intracerebral hemorrhage patient with augmented renal clearance. Eur J Clin Pharmacol. 2018;74(8):1091-1092.

33. Martínková J, Malbrain ML, Havel E, Šafránek P, Bezouška J, Kaška M. A pilot study on pharmacokinetic/pharmacodynamic target attainment in critically ill patients receiving piperacillin/tazobactam. Anaesthesiol Intensive Ther. 2015;48(1):23-30.

34. Patel PR, Cook SE. Stability of meropenem in intravenous solutions. Am J Health Syst Pharm. 1997;54(4):412-421.

35. Tomasello C, Leggieri A, Cavalli R, Di Perri G, D'Avolio A. In vitro stabilifighty evaluation of different pharmaceutical products containing meropenem. Hosp Pharm. 2015;50(4):296-303.

36. Katip W, Wientong P, Sornsuvit C. The stability of generic meropenem in tropical countries. Int J Pharm Pharm Sci. 2015;7(1):236-238.

37. Franceschi L, Cojutti P, Baraldo M, Pea F. Stability of generic meropenem solutions for administration by continuous infusion at normal and elevated temperatures. Ther Drug Monit. 2014;36(5):674-669.

38. Roberts DM, Roberts JA, Roberts MS, et al; RENAL Replacement Therapy Study Investigators. Variability of antibiotic concentrations in critically ill patients receiving continuous renal replacement therapy: A multicentre pharmacokinetic study. Crit Care Med. 2012;40(5): 1523-1528. 